commercially available AKT and DNAPK inhibitors with cisplatin, and elucidate their mechanism of action within the PI3K/ $\mathrm{AKT} / \mathrm{mTOR}$ pathway.

Methodology Platinum resistant immortalised HGSOC cell lines (PEO4, PEA2, OVCAR8, Kuramochi) were treated with cisplatin plus/minus AKT or DNA-PK inhibitors and Isobologram assays performed to establish synergy/antagonism between drug treatments. Cells were treated with inhibitors plus/minus cisplatin at different time points, protein lysates collected, and Reverse Phase Protein Array (RPPA) proteomics performed and analysed to establish mechanisms of action of inhibitors on the PI3K/AKT/mTOR pathway.

Result(s)* Following treatment with cisplatin in combination with AKT or DNA-PK inhibitors, different levels of synergy were observed in platinum resistant HGSOC cell lines; strong synergy was noted for AKT inhibitors Afurosertib, Uprosertib, and Triciribine. Proteomic analysis revealed a response signature for AKT or DNAPK inhibition showing activation of AKT at S473 and decrease of downstream targets pS6 235/ 236 and 240/44, and p70S6K_T389.

Conclusion* In the platinum resistant immortalised HGSOC cell lines tested, AKT inhibitors showed a synergistic effect when used in combination with cisplatin. Proteomic analysis confirmed targeting of the PI3K/AKT/mTOR pathway. With the aim of resensitising a resistant patient to their platinumbased chemotherapy a synergistic effect between the resensitising compound and chemotherapy agent is essential; this data suggests targeting of the PI3K/AKT/mTOR pathway in platinum-resistant HGSOC patients with AKT or DNAPK inhibition is a potentially useful therapeutic strategy.

\section{IMPLEMENTATION AND FEASIBILITY OF PROPHYLACTIC BILATERAL SALPINGECTOMY AT BENIGN, MINIMALLY INVASIVE HYSTERECTOMY IN STYRIA (AUSTRIA)}

${ }^{1} \mathrm{C}$ Hütter, ${ }^{1} \mathrm{~K}$ Tamussino*, ${ }^{2} \mathrm{~K}$ Simon, ${ }^{3} \mathrm{M}$ Kratky, ${ }^{4} \mathrm{C}$ Mutz-Eckhart, ${ }^{5} \mathrm{M}$ Blatt-Gunegger, ${ }^{5} \mathrm{~S}$ Klammer, ${ }^{6,7} \mathrm{C}$ Bermann, ${ }^{8} \mathrm{~A}$ Huber, ${ }^{9} \mathrm{~V}$ Lessiak. ${ }^{1}$ Medical University Graz, Gynecology, Graz, Austria; 'Landeskrankenhaus Hochsteiermark, Standort Leoben, Leoben, Austria; ${ }^{3}$ Landeskrankenhaus Weststeiermark, Standort Deutschlandsberg, Deutschlandsberg, Austria; ${ }^{4}$ Landeskrankenhaus Murtal, location Judenburg, Judenburg, Austria; ${ }^{5}$ LKH Rottenmann - Bad Aussee, Bad Aussee, Austria; ${ }^{6}$ Lkh Hartberg, Hartberg, Austria; ${ }^{7}$ Medical University Graz; ${ }^{8}$ Lkh Feldbach, Feldbach, Austria; ${ }^{9}$ Krankenhaus der Barmherzigen Brüder Graz, Standort Marschallgasse, Graz, Austria

\subsection{6/ijgc-2021-ESG0.397}

Introduction/Background* Numerous societies, including the Austrian Society of Obstetrics \& Gynecology (OEGGG) in 2015, have recommended prophylactic bilateral salpingectomy (PBS) at the time of benign gynecologic surgery with the intent of ovarian cancer risk reduction. We evaluated implementation and feasibility of PBS at benign, minimally invasive hysterectomy in public hospitals in the Austrian province of Styria in 2014 vs. 2018 (before and after the official recommendation in 2015).

Methodology We reviewed surgical consent forms and operative notes of patients undergoing vaginal or laparoscopic hysterectomy for benign indications in Styria in 2014 and 2018. Ethics approval was obtained.

Result(s)* 1,256 benign, minimally invasive hysterectomies were identified (580 in 2014, 676 in 2018). 68\% of patients were consented for PBS in 2014 and 94\% in 2018 (P < $0.05)$. The PBS rate in consented patients was $88 \%$ in 2014 and $83 \%$ in 2018 (n.s.). In 2018 PBS was completed more often at laparoscopic than at vaginal hysterectomy (95\% vs $74 \%, \mathrm{P}<0.05)$. Age and parity were the major influencing factors for success of PBS.

Conclusion* PBS at minimally invasive hysterectomy was widely performed in Styria even before the official recommendation in 2015, and increased thereafter to $83 \%$ overall in 2018. PBS was accomplished somewhat more often at laparoscopic than at vaginal hysterectomy.

\section{ANALYSIS OF THE CLINICAL EXPERIENCE WITHIN RUCAPARIB'S EARLY ACCESS PROGRAM IN SPAIN - A GEICO STUDY}

${ }^{1} A$ Yubero-Esteban*, ${ }^{2} A$ Barquin, ${ }^{3} P$ Estévez, ${ }^{4} B$ Pajares Hachero, ${ }^{5} \mathrm{~L}$ Sánchez, ${ }^{6} \mathrm{P}$ Reche, ${ }^{7} \mathrm{JD}$ Alarcón, ${ }^{8} \mathrm{~J}$ Calzas, ${ }^{9} \mathrm{~L}$ Gaba, ${ }^{10} \mathrm{~J}$ Fuentes-Pradera, ${ }^{11} \mathrm{~A}$ Santaballa, ${ }^{12} \mathrm{C}$ Salvador, ${ }^{13} \mathrm{~L}$ Manso, ${ }^{14} \mathrm{~A}$ Herrero-lbáñez, ${ }^{15} \mathrm{~A}$ Taus, ${ }^{16} \mathrm{R}$ Márquez, ${ }^{17} \mathrm{~J}$ Madani, ${ }^{18} \mathrm{M}$ Merino, ${ }^{19} \mathrm{G}$ Marquina, ${ }^{5} \mathrm{~A}$ González-Martín. ${ }^{1}$ Hospital Clínico Universitario Lozano Blesa, Medical Oncology, Zaragoza, Spain; ${ }^{2}$ Centro Integral Oncológico Clara Campal, Medical Oncology, Madrid, Spain; ${ }^{3}$ Hospital Universitario Virgen del Rocío, Medical Oncology, Sevilla, Spain; ${ }^{4}$ Hospital Universitario Virgen de la Victoria, Medical Oncology, Málaga, Spain; ${ }^{5}$ Clínica Universidad de Navarra, Medical Oncology, Madrid, Spain; ${ }^{6}$ Hospital Universitario Torrecárdenas, Medical Oncology, Almería, Spain; ${ }^{7}$ Hospital Universitari Son Espases, Medical Oncology, Palma, Spain; ${ }^{8}$ Hospital Universitario de Fuenlabrada, Medical Oncology, Fuenlabrada, Spain; ${ }^{9}$ Hospital Clínic de Barcelona, Medical Oncology, Barcelona, Spain; ${ }^{10}$ Hospital Universitario Virgen de Valme, Medical Oncology, Sevilla, Spain; ${ }^{11}$ Hospital Universitari i Politècnic la Fe, Medical Oncology, Valencia, Spain; ${ }^{12}$ Hospital Lluis Alcanyís de Xàtiva, Medical Oncology, Xàtiva, Spain: ${ }^{13}$ Hospital Universitario 12 de Octubre, Medical Oncology, Madrid, Spain; ${ }^{14}$ Hospital Universitario Miguel Servet, Medical Oncology, Zaragoza, Spain; ${ }^{15} \mathrm{Hospital}$ del Mar, Medical Oncology, Barcelona, Spain; ${ }^{16} \mathrm{MD}$ Anderson Cancer Center Madrid, Medical Oncology, Madrid, Spain; ${ }^{17}$ Hospital General San Jorge, Medical Oncology, Huesca, Spain; ${ }^{18}$ Hospital Universitario Infanta Sofía, Medical Oncology, Madrid, Spain; ${ }^{19}$ Hospital Clínico San Carlos, Medical Oncology, Madrid, Spain

\subsection{6/ijgc-2021-ESG0.398}

Introduction/Background* Rucaparib is a PARP-1/2/3 inhibitor approved for the treatment of high-grade ovarian cancer (HGOC). In ARIEL3, rucaparib improved PFS as maintenance therapy for platinum $(\mathrm{Pt})$-sensitive recurrent OC. Study 10, ARIEL2, and ARIEL4 showed rucaparib's benefit as treatment. An observational study was performed in HGOC pts treated within the rucaparib access program (RAP) in Spain. The aim was to better understand rucaparib's management in real-life setting, to optimize future use, considering Pt-sensitive and Pt-resistant BRCAmut treatment and maintenance patients.

Methodology A retrospective study was performed at 22 GEICO hospitals in Spain that treated pts within RAP (600 mg BID) since September 2018. Adult women with high-grade epithelian ovarian, fallopian tube, or primary peritoneal cancer, with medical record available, were included. Patient characteristics, medical history, safety, efficacy, and dosing data were collected.

Result(s)* Between July 2020 and February 2021, 51 pts were recruited with median age 63 years (36-86). At diagnosis, $45.1 \%$ of patients harbored gBRCA mutations, $19.6 \%$ sBRCA mutations, and 31.4\% were BRCAwt. Before rucaparib, pts had ECOG PS 0,1 , or $2(37.3 \%, 49.0 \%$, and $5.9 \%)$ and $72.5 \%$ had measurable disease. The median number of previous lines was 4 (1-9), 51.0\% of pts received prior bevacizumab, and notably $25.5 \%$ of pts had received a prior PARPi. Rucaparib was given as maintenance, Pt-resistant, and Pt-sensitive treatment in $35.3 \%, 51.0 \%$, and $13.7 \%$ of pts respectively (median dose $557.7 \mathrm{mg}[300-600]$ ). $82.4 \%$ of pts received rucaparib for $\leq 12 \mathrm{mo}$ and $17.6 \%>12$ mo. $50.0 \%$ 